\section{Probability inequalities for testing separate activation models of divided attention}

\author{
ADELE DIEDERICH \\ C. v. Ossietzky Universitat Oldenburg \\ Oldenburg, Germany
}

A test for separate activation models of reaction time proposed by Miller (1982) is generalized to apply to situations with more than two redundant targets.

Traditionally, perception and information processing are studied in one single sensory modality, isolated from all remaining modalities. For example, in the determination of hearing thresholds, the influence of other modalities such as vision or feeling is to be eliminated as much as possible. From time to time, psychologists have been interested in the effects on detection or recognition when different modalities are combined. Welch and Warren (1986) use the term intersensory interaction to designate the situation in which the perception of an event as measured in terms of one sensory modality is changed in some way by the concurrent stimulation of one or more other sensory modalities. Intersensory interaction may be called intersensory facilitation or simply facilitation, for short, if the interaction results in faster processing at some stages of processing-that is, by reducing reaction time-or if it improves the perceptibility of stimuli (e.g., by lowering sensory thresholds). Here we consider experimental paradigms that result in a faster response when two or more targets are present than when only one target is present. A similar effect occurs when two or more targets are presented within one modality (see, e.g., van der Heijden, 1975). It should be noted that all our formal results presented below apply to this redundant target situation as well.

Today, essentially two different approaches have been suggested to explain the phenomenon of intersensory facilitation: coactivation models and separate activation models (Miller, 1982). Separate activation models assume that processing in different sensory channels occurs in parallel. Presenting multiple stimuli (e.g., light and tone or light, tone and vibration) produces separate activation in each of the corresponding channels. Either activation builds to the level at which it can produce a response. This means that the response is produced by the signal that is processed first. These models are also called race models, since a race between several response activation processes takes place and the winner determines the re-

Thanks are due the reviewers, Jeff Miller and Toby Mordkoff, for some helpful comments on an earlier version of this note that improved the presentation of the results. Address correspondence to A. Diederich, Fachbereich Physik, Universität Oldenburg, P.O. Box 2503, D-2900 Oldenburg, Germany. sponse. Separate activation models predict faster average reaction to multiple stimuli than to single stimuli, since the average of the minimum of the processing times in different channels is smaller than the average processing time in either channel, provided that we assume processing times to be random variables, thus:

$$
\mathrm{RT}_{\mathrm{VA}}=\min \left(\mathrm{RT}_{\mathrm{V}}, \mathrm{RT}_{\mathrm{A}}\right) \text {, }
$$

where $\mathrm{RT}_{\mathrm{VA}}$ denotes the random reaction time to a double stimulus, when a visual (V) and an auditory (A) stimulus are presented together, whereas $\mathrm{RT}_{\mathbf{v}}$ and $\mathrm{RT}_{\mathrm{A}}$ denote the reaction times to a visual and to an auditory stimulus, respectively, when these stimuli are presented alone.

An alternative approach to explain intersensory facilitation is coactivation. Coactivation models assume that interaction between different sensory modalities is possible. When multiple stimuli are presented, activation of different channels can be combined to satisfy a single criterion for response initiation. Coactivation models predict faster average reaction time to multiple stimuli compared to single stimuli because the combined activation reaches this criterion faster.

A currently popular test for separate activation was proposed by Miller (1982). The test is based on a prediction of the separate activation assumption for reaction time distributions. We derive it here in a general context of probability inequalities that allow the derivation of further tests when more than two modalities are involved.

\section{A Generalized Test For Separate Activation}

Let $A_{1}, \ldots, A_{m}$ be $m$ events in some probability space. Then the principle of inclusion and exclusion gives the well-known Poincare formula for the probability of occurrence of at least one of the events (Chung, 1979):

$$
\begin{aligned}
P\left(\bigcup_{i=1}^{m} A_{i}\right)=\sum_{i=1}^{m} P\left(A_{i}\right) & -\sum_{i<j} P\left(A_{i} A_{j}\right)+\sum_{i<j<k} P\left(A_{i} A_{j} A_{k}\right) \\
& +\ldots+(-1)^{m-1} P\left(A_{1} A_{2} \ldots A_{m}\right)
\end{aligned}
$$

with $m \geq 1, A_{i} A_{j}=A_{i} \cap A_{j}$, and so forth. Moreover, the sum of the first $P$ terms on the right hand side of Equation 2 provides an upper bound for the left side if $P$ is odd and a lower bound if $P$ is even. Thus we get a sequence of inequalities of which the first are

$$
P\left(\bigcup_{i=1}^{m} A_{i}\right)-\sum_{i<j} P\left(A_{i} A_{j}\right) \leq P\left(\bigcup_{i=1}^{m} A_{i}\right) \leq \sum_{i=1}^{m} P\left(A_{i}\right), m=2,3, \ldots
$$

The left hand side of Equation 3 is called Bonferroni inequality, whereas the right hand side of Equation 3 is referred to as Boole's inequality. Since $\Sigma_{i} P\left(A_{i}\right)$ can exceed 1 , the upper bound is strictly $\min \left[\Sigma_{i} P\left(A_{i}\right), 1\right]$; for the lower bound, negative values can be replaced by 0 . For notational convenience, this is not made explicit in 
the equation above. Usually the upper bound is more important because it provides a conservative test, but it is not as accurate as the lower bound (Worsley, 1982).

Identifying $A_{i}$ with the event $\left(X_{i} \leq x\right)$ will then yield

$$
P\left(\bigcup_{i=1}^{m} A_{i}\right)=P\left[\min \left(X_{i}: i=1, \ldots, m\right) \leq x\right]
$$

for a sequence of random variables $X_{i}, i=1, \ldots, m$ on a given probability space. As mentioned above, the race mechanism in the model of separate activation postulates such that in the double stimulus situation detection time is determined by the minimum of the two stimulus processes

$$
R T_{V_{A}}=\min \left(R T_{V}, R_{A}\right)
$$

Therefore,

$$
P\left[\min \left(\mathrm{RT}_{\mathrm{v}}, \mathrm{RT}_{\mathrm{A}}\right) \leq t\right] \leq P\left(\mathrm{RT}_{\mathrm{v}} \leq t\right)+P\left(\mathrm{RT}_{\mathrm{A}} \leq t\right),
$$

for any $t$. As observed by Miller (1982), Equation 5 is a prediction of all separate activation models; the inequality puts an upper bound on the facilitation produced by double stimuli. All separate activation models can be rejected if observed facilitation is more than that consistent with the inequality-that is, if the inequality is violated for any value of $t$. On the other hand, nonviolation of Equation 5 does not represent evidence in favor of separate activation models, since coactivation mechanisms satisfying it are conceivable (Colonius, 1986, 1990; Diederich, 1985, 1987; Diederich \& Colonius, 1987, 1991; Miller, 1982; Ulrich \& Giray, 1986). Moreover, Eriksen (1988) has pointed out that fast guesses can strongly bias this test in favor of separate activation (cf. Miller \& Lopes, 1991).

For illustrative purposes, consider an experiment in which stimuli of three different modalities are presented-for example, visual, auditory, and tactile. Let $\mathrm{RT}_{\mathrm{T}}, \mathrm{RT}_{\mathrm{V}}$, and $\mathrm{RT}_{\mathrm{A}}$ denote the reaction time random variable in the tactile, visual, and auditory stimulus conditions, respectively, and $\mathrm{RT}_{\mathrm{TVA}}$ the reaction time random variable in the triple stimulus condition. For the triple stimulus condition, then, we get

$$
\begin{aligned}
& P\left(\mathrm{RT}_{\mathrm{TVA}_{\mathrm{A}}} \leq t\right) \\
& \quad=P\left[\min \left(\mathrm{RT}_{\mathrm{T}}, \mathrm{RT}_{\mathrm{V}}, \mathrm{RT}_{\mathrm{A}} \leq t\right)\right] \\
& \quad \leq P\left(\mathrm{RT}_{\mathrm{T}} \leq t\right)+P\left(\mathrm{RT}_{\mathrm{V}} \leq t\right)+P\left(\mathrm{RT}_{\mathrm{A}} \leq t\right) .
\end{aligned}
$$

This inequality puts an upper bound on the facilitation produced by triple stimuli, but unfortunately, this bound is likely to be too conservative to be of much use for testing for separate activation. Interestingly, there are various ways of sharpening the upper bound in Equation 3, such as

$$
P\left(\bigcup_{i=1}^{m} A_{i}\right) \leq \sum_{i=1}^{m} P\left(A_{i}\right)-\sum_{i=1}^{m-1} P\left(A_{i}, A_{i+1}\right)
$$

For a proof of this and related results, the reader is referred to Worsley (1982).

For our special case with $m=3$, we get

$$
\begin{aligned}
& P\left(A_{1} \cup A_{2} \cup A_{3}\right) \\
& \leq P\left(A_{1}\right)+P\left(A_{2}\right)+P\left(A_{3}\right)-\left[P\left(A_{1}, A_{2}\right)+P\left(A_{2}, A_{3}\right)\right] \\
&= P\left(A_{1}+P\left(A_{2}\right)+P\left(A_{3}\right)\right. \\
& \quad-P\left(A_{1}\right)+P\left(A_{2}\right)-P\left(A_{1} \cup A_{2}\right) \\
&\left.+P\left(A_{2}\right)+P\left(A_{3}\right)-P\left(A_{2} \cup A_{3}\right)\right] \\
&= P\left(A_{1} \cup A_{2}\right)+P\left(A_{2} \cup A_{3}\right)-P\left(A_{2}\right)
\end{aligned}
$$

Thus, we have a sharper upper bound for the facilitation produced by the triple stimulus condition. By symmetry, this yields three different inequalities:

$$
\begin{aligned}
P & {\left[\min \left(\mathrm{RT}_{\mathrm{T}}, \mathrm{RT}_{\mathrm{V}}, \mathrm{RT}_{\mathrm{A}}\right) \leq t\right] } \\
\leq & P\left[\min \left(\mathrm{RT}_{\mathrm{T}}, \mathrm{RT}_{\mathrm{V}}\right) \leq t\right]+P\left[\min \left(\mathrm{RT}_{\mathrm{V}}, \mathrm{RT}_{\mathrm{A}}\right) \leq t\right] \\
& -P\left(\mathrm{RT}_{\mathrm{V}} \leq t\right)
\end{aligned}
$$

or

$$
\begin{aligned}
& P\left[\min \left(\mathrm{RT}_{\mathrm{T}}, \mathrm{RT}_{\mathrm{V}}, \mathrm{RT}_{\mathrm{A}}\right) \leq t\right] \\
& \leq P\left[\min \left(\mathrm{RT}_{\mathrm{T}}, \mathrm{RT}_{\mathrm{V}}\right) \leq t\right]+P\left[\min \left(\mathrm{RT}_{\mathrm{T}}, \mathrm{RT}_{\mathrm{A}}\right) \leq t\right] \\
& \quad-P\left(\mathrm{RT}_{\mathrm{T}} \leq t\right)
\end{aligned}
$$

or

$$
\begin{aligned}
P & {\left[\min \left(\mathrm{RT}_{\mathrm{T}}, \mathrm{RT}_{\mathrm{V}}, \mathrm{RT}_{\mathrm{A}}\right) \leq t\right] } \\
\leq & P\left[\min \left(\mathrm{RT}_{\mathrm{T}}, \mathrm{RT}_{\mathrm{A}}\right) \leq t\right]+P\left[\min \left(\mathrm{RT}_{\mathrm{V}}, \mathrm{RT}_{\mathrm{A}}\right) \leq t\right] \\
& -P\left(\mathrm{RT}_{\mathrm{A}} \leq t\right) .
\end{aligned}
$$

Note that all these RT distributions are observable: the RT when all three stimuli are present; the RT when any two stimuli are present; and the RT when just one stimulus is present. Moreover, there is a further sharpening of the upper bound by taking, for each $t$, the minimum of all three upper bounds.

These inequalities can also be used for data from an experiment where in the double stimulus situation the stimuli are $\tau$ milliseconds apart and in the triple stimulus situation the second stimulus is presented $\tau_{1}$ milliseconds later than the first and the third stimulus is presented $\tau_{2}$ milliseconds later than the first stimulus. For this situation we have

$$
\begin{aligned}
P\left(\mathrm{RT}_{\mathrm{TVA}\left(\tau_{1}, \tau_{2}\right)} \leq t\right) & \\
= & P\left[\min \left(\mathrm{RT}_{\mathrm{T}}, \mathrm{RT}_{\mathrm{V}+\tau_{1}}, \mathrm{RT}_{\mathrm{A}+\tau_{2}}\right) \leq t\right] \\
\leq & P\left[\min \left(\mathrm{RT}_{\mathrm{T}}, \mathrm{RT}_{\mathrm{V}+\tau_{1}}\right) \leq t\right] \\
& +P\left[\min \left(\mathrm{RT}_{\mathrm{V}+\tau_{1}}, \mathrm{RT}_{\mathrm{A}+\tau_{2}}\right) \leq t\right]-P\left(\mathrm{RT}_{\mathrm{V}+\tau_{1}} \leq t\right)
\end{aligned}
$$

Our empirical results on these tests indicate serious violations of the separate activation assumption with stimuli from three different modalities (see Diederich, 1992). 


\section{REFERENCES}

Chung, K. L. (1979). Elementary probability theory with stochastic processes. Berlin: Springer-Verlag.

Colonius, H. (1986). Measuring channel dependence in separate activation models. Perception \& Psychophysics, 40, 251-255.

Colonius, H. (1990). Possibly dependent probability summation of reaction time. Journal of Mathematical Psychology, 43, 253-275.

DiEderich, A. (1985). Eine experimentelle und theoretische Analyse der intersensorischen Bahnung. Unpublished diploma thesis, Oldenburg University.

DIEDERICH, A. (1987). Measuring facilitation in the motor component. In E. E. Roskam \& R. Suck (Eds.), Progress in mathematical psychology I (pp. 209-217). Amsterdam: North-Holland.

DIEDERICH, A. (1992). Intersensory facilitation with multiple stimuli: Race, superposition, and diffusion models for reaction time. Frankfurt: Peter Lang.

Diederich, A., \& Colonius, H. (1987). Intersensory facilitation in the motor component? A reaction time analysis. Psychological Research, 49, 23-29.

Diederich, A., \& Colonius, H. (1991). A further test of the superposition model for the redundant-signals effect in bimodal detection. Perception \& Psychophysics, 50, 83-86.
ErIKSEN, C. W. (1988). A source of error in attempts to distinguish coactivation from separate activation in the perception of redundant targets. Perception \& Psychophysics, 44, 191-193.

MilleR, J. (1982). Divided attention: Evidence for coactivation with redundant signals. Cognitive Psychology, 14, 247-279.

Miller, J., \& LOPES, A. (1991). Bias produced by fast guessing in distribution-based tests of race models. Perception \& Psychophysics, 50, 584-590.

ULRICH, R., \& GIRAY, M. (1986). Separate-activation models with variable base times: Testability and checking of cross-channel dependency. Perception \& Psychophysics, 39, 248-254.

van der Heijden, A. H. C. (1975). Some evidence for a limited capacity parallel self-terminating process in simple visual search tasks. Acta Psychologica, 39, 21-41.

WELCH, R. B., \& WARREN, D. V. (1986). Intersensory interaction. In K. R. Boff, L. Kaufman, \& J. P. Thomas (Eds.), Handbook of perception and human performance (Vol. 1, pp. 25-1 to 25-35). San Diego: Academic Press.

WORSLEY, K. J. (1982). An improved Bonferroni inequality and applications. Biometrika, 69, 297-302.

(Manuscript received March 12, 1992; revision accepted for publication May 26, 1992.) 